\title{
An Economical Frequency Synthesizer Using Interpolation Techniques
}

\author{
David Shiung and Huei-Wen Ferng† \\ Novatek Mocroelectronics Corp. \\ 2F, No. 13, Innovation Road I, Science-Based Industrial Park, Hsin-Chu, 300, Taiwan \\ E-mail: david_shiung@ novatek.com.tw \\ $\dagger$ Department of Computer Science and Information Engineering \\ National Taiwan University of Science and Technology, Taipei 106, Taiwan \\ E-mail: hwferng@mail.ntust.edu.tw
}

\begin{abstract}
In this paper, we propose an all-digital frequency synthesizer using interpolation techniques. Unlike the traditional direct digital synthesizer (DDS) which requires a large look-up table to store the phase and its corresponding sine wave, our new design contains only a linear feedback shift register (LFSR) and two banks of memory to store the two reference sequences. This can achieve a cost-efficient design. The novelty of this idea lies in that one can synthesize the desired frequency between these two reference frequencies through a threshold device. The frequency resolution of this synthesizer is determined by the order of the LFSR. The maximal spur around the synthesized frequency may be controlled by the numerical precision of these two reference frequencies. The percentage error of the synthesized frequency is identical to that of the system clock. This design is mainly used in implementing an economical digital modulator for continuous phase frequency shift keying (CPFSK) and other related wireless communication arts.
\end{abstract}

\section{INTRODUCTION}

Accompanying the rapid development of complementary metal-oxide semiconductor (CMOS) fabrication techniques, digital design has become more and more popular in the recent few years. Unlike its analog counterpart, digital circuitry deserves much more robustness against fabrication variation and more efficient in power consumption. Besides, digital circuits are relatively insensitive to the change of temperature, pressure, and aging [2]. These effects have driven the development of digital circuitry profoundly.

Regarding the research interest of wireless communications, frequency synthesizers always play a key role in designing a communication system. Typically, these applications may include frequency modulation/demodulation of the transmitter and receiver, respectively [8], [9]. For these wireless communication applications, system designers should carefully use the system resources and follow the related regulations. These scanty resources may be, for example, bandwidth. An accurate and precise frequency synthesizer is thus essential in designing a wireless transceiver. Among all the alreadyknown frequency synthesis techniques that suited for the very large scale integrated (VLSI) design, phase locked loop (PLL) are widely used in today's commerical products. These PLLs may be all-digital or a mixed-signal device that depend on the system requirements [1]. However, a PLL-based design sometimes is not a cost-effective choice.

Besides this traditional technique, there still exists other frequency synthesizers, e.g., DDS. Spotting on the various techniques, a comprehensive comparison is reported in [4]. Regarding these merits and drawbacks of these possible candidates, digital synthesizers often reveal much faster tuning speed and settling time than their analog competitors. Besides, a digital synthesizer is often easy to generate a much higher frequency resolution.

In this paper, an all-digital frequency synthesizer that aims at providing a hardware-efficient architecture is proposed. In this new architecture, only two sets of memory banks and some other simple logic are used. These two reference frequencies are predetermined according to the system specifications. Except for these two memory banks, our new design also contains a LFSR. Conceptually our new design can interpolate all the frequencies between these two reference frequencies. We may conveniently call this new synthesizer interpolation synthesizer (IS). Since this new design is fully digital, it almost inherits all the benefits of a DDS such as high frequency resolution, fast settling time, and etc. The synthesized frequency is determined by the threshold of the threshold device. If the value of LFSR is less than a predetermined threshold, the output sequence should be, say, sequence 1; on the other hand, if this value is larger than the threshold, the output sequence will be sequence 2. The novelty of this idea lies in that it is not necessary to build a large look-up table (LUT) for each phase increment as a DDS. Although some approaches to reduce the size of a DDS have been proposed, e.g., [3] and [10], it is still hardware-expensive to implement this block. In our design, analog waveform may also be generated if a digital-to-analog converter (DAC) is cascaded behind the IS.

The remaining parts of this paper are organized as follows. In Sec. II, we present the system blocks of this synthesizer. In Sec. III, we present the numerical results to examine the accuracy of synthesized frequency and impact of finiteprecision effect. Finally, conclusions are concluded in Sec. IV. 


\section{SYSTEM DIAGRAM OF IS}

The system blocks of the IS are shown in Fig. 1. This synthesizer mainly consists of two memory banks that store two reference sequences with frequencies $f_{1}$ and $f_{2}$ and a LFSR to determine the output sequence. The system clock rate is denoted as $f_{s}$. The system clock period $T_{s}$ may be written as

$$
T_{s}=\frac{1}{f_{s}} \text {. }
$$

Since the output sample rate is fixed, the lengths of these two sequences should be different from each other. These two reference sequences may be represented as

$$
\begin{gathered}
x_{1}[n]=\sin \left(2 \pi f_{1}\left(n_{1}-1\right) T_{s}\right) \\
x_{2}[n]=\sin \left(2 \pi f_{2}\left(n_{2}-1\right) T_{s}\right),
\end{gathered}
$$

where $n_{1}=1, \cdots, N_{1}$ and $n_{2}=1, \cdots, N_{2} . N_{1}$ and $N_{2}$ resemble the periods of sequences $x_{1}[n]$ and $x_{2}[n]$, respectively. Notice that we should choose $f_{1}$ and $f_{2}$ as close as possible so as to generate a pure frequency. We may represent $N_{1}$ and $N_{2}$ as

$$
N_{1}=\frac{f_{s}}{f_{1}}
$$

and

$$
N_{2}=\frac{f_{s}}{f_{2}}
$$

We may use these two sequences $x_{1}[n]$ and $x_{2}[n]$ to synthesize the intended signal with frequency $f_{\text {sync. }}$. The method of generating $f_{\text {sync }}$ comes from the idea that the synthesized frequency may be constituted by two sinusoids with quasirandom probability. The minimal frequency that can be resolved is determined by the order of the LFSR. A trace of this idea may also be found in a fractional- $\mathrm{N}$ frequency synthesizer [7]. By assigning different probability to the reference signal, one can change the constituting components of the output sequence. Intuitively $f_{\text {sync }}$ should lie between $f_{1}$ and $f_{2}$. In order to obtain a stable output, the difference between these two reference frequencies should be as small as possible.

Regarding the design of a LFSR, there are basically two configurations to generate a maximal length sequences; namely, Galois feedback generator and Fibonacci feedback generator [6]. These two generators are functionally equivalent and are with identical hardware complexity. We may arbitrarily choose Galois feedback generator in our new design. We reproduce this configuration in Fig. 2. The frequency resolution of this synthesizer is determined by the order of the LFSR $r$ and the characteristics of a LFSR is determined by the generating polynomial

$$
g(D)=\sum_{i=0}^{r} g_{i} D^{i}
$$

If $g(D)$ is primitive, the outcome of the LFSR $\left(a_{r-1}, a_{r-2}, a_{r-3}, \ldots, a_{2}, a_{1}, a_{0}\right)$ is with a period $2^{r}-1$ and is called degenerated [6]. In order to achieve a better frequency resolution, a primitive polynomial is recommended.
For the reason of simplicity in notation representation, we assume that $f_{2}>f_{1}$ and the equivalent value of the LFSR lies between $[0,1)$. If this value is less than a predetermined threshold $\mathrm{TH}$, the output sequence is $x_{2}[n]$; otherwise, the output is $x_{1}[n]$. We may write $f_{\text {sync }}$ as

$$
f_{\text {sync }}=T H \cdot f_{2}+(1-T H) \cdot f_{1} .
$$

If the generating polynomial of the LFSR is primitive, all the $2^{r}-1$ transition states will be different. Thus, one yields the frequency resolution

$$
f_{\text {res }}=\frac{f_{2}-f_{1}}{2^{r}-1}
$$

Substitute (4) and (5) into (7), we get

$$
f_{\text {sync }}=\left(\frac{T H}{N_{2}}+\frac{1-T H}{N_{1}}\right) \cdot f_{s}
$$

According to the sampling theorem, the maximal achievable frequency is limited to half the system clock rate [3], [7]. In addition, the synthesized frequency is with identical percentage error as the system clock if there exists a frequency error of the system clock. This maybe proved by extending (9). The methodology of how to quantize a real number into a finite-precision one is also an important issue for a digital frequency synthesizer. We will touch upon this issuein the following section.

\section{NUMERICAL RESUlTS}

In this section, we provide two examples to examine the effectiveness of the newly proposed frequency synthesizer. The octal representation of the generating polynomial used in these two examples are [2011] and the initial setting of the registers in LFSR are all 1's.

In Figs. 3-4, we use the method of averaging periodogram [5] to examine both the correctness and the impact of finite word-length effect on the power spectral density (PSD) of the synthesized frequency. The PSDs are each estimated by averaging 61 periodograms each with length 26784 and zoomed into appropriate frequency bands. The frequency resolution in these two examples is $1(\mathrm{kHz})$. The sampling frequency $f_{s}$ used here is $26.784(\mathrm{MHz})$ and the intended frequency is $201.008(\mathrm{kHz})$. We thus may choose $N_{1}=134, N_{2}=133$, and $T H=0.75$. Although there still exists other candidates for $N_{1}, N_{2}$, and $\mathrm{TH}$, two neighboring reference frequencies may yield better performance in phase noise. In fig. 3, we use floating-point format to resemble Seqs. 1 and 2. Also shown in Fig. 3 is a sinusoidal signal with an exact frequency 201.008 $(\mathrm{kHz})$. Note that both curves in Fig. 3 are normalized to its peak amplitude for fair comparison. Evidently, both curves are with identical center frequency. The phase noise at a 100 $(\mathrm{kHz})$ offset is approximately $-67.2-10 \log _{10} 10^{3}=-97.2$ $(\mathrm{dBc} / \mathrm{Hz})$.

In Fig. 4, we consider the impact of finite-precision numerical effect on the spectra of synthesized signals. Typically, we use 5 bits to represent each sample of Seqs. 1 and 2 . Conceptually finite-precision effect causes spurs at higher 
frequency band. These spurs may be mitigated if we allocate more bits to represent the reference frequencies. The maximal spur in this case is located around $1000(\mathrm{kHz})$ and the frequency suppression of the maximal spur is approximately $47.8 \mathrm{~dB}$.

\section{CONCLUSIONS}

In this paper, we propose an economical frequency synthesizer. Unlike a traditional DDS that uses a large memory to save all the possible output samples, only two reference sequences are kept. This design may be implemented using VLSI and may be fabricated using CMOS fabrication process. Both digital and analog output are available in our design. As compared to its analog competitor, a much faster settling time may be achieved. This new design also inherits almost all the benefits of digital circuitry. Theoretically, the highest frequency for a digital synthesizer is half the system clock rate. However, if we intend to get a better phase-noise performance, the highest achievable frequency should be less than $\frac{f_{s}}{3}$.

\section{REFERENCES}

[1] J. Dunning, G. Garcia, J. Lundberg, and E. Nuckolls, "An Alldigital Phase-locked Loop with 50-cycle Lock Time Suitable for Highperformance Microprocessors," IEEE Journal of Solid-state Circuits, vol. 30, no. 4, pp. 412-422, April 1995.

[2] E. Grayver and B. Daneshrad, "A Low-power All-digital FSK Receiver for Space Applications," IEEE Trans. Commun., vol. 49, no. 49, pp. 911921 , May 2001.

[3] H. T. Nicholas, III and H. Samueli, "A 150-MHz Direct Digital Frequency Synthesizer in $1.25-\mu \mathrm{m}$ CMOS with $-90-\mathrm{dBc}$ Spurious Performance," IEEE J. Solid-State Circuits, vol. 26, no. 12, pp. 1959-1969, Dec. 1991.

[4] D. P. Noel and T. A. Kwasniewski, "Frequency Synthesis: A Comparison of Techniques," Proc. 1994 Canadian Conference on Electrical and Computer Engineering, NS Canada, pp. 535-538, Sep. 25-28, 1994.

[5] A. V. Oppenheim, R. W. Schafer, and J. R. Buck, Discrete-time Signal Processing, Englewood Cliffs, NJ: Prentice-Hall, 1999.

[6] R. L. Peterson, R. E. Ziemer, and D. E. Borth, Introduction to Spread Spectrum Communications, Englewood Cliffs, NJ: Prentice-Hall, 1997.

[7] B. Razavi, RF Microelectronics, Englewood Cliffs, NJ: Prentice-Hall, 1998.

[8] R. Uusikartano and J. Niittylahti, "A Digital Frequency Synthesizer for a 2.4 GHz Fast Frequency Hopping Transceiver," Proc. 43rd IEEE Midwest Symp. on Circuits and Systems, Lansing MI, 2000, pp. 420-423, Aug. 811.

[9] J. Vankka, "Digital Frequency Synthesizer/Modulator for Continuousphase Modulations with Slow Frequency Hopping," IEEE Trans. Vehicular Tech., vol. 46, no. 4, pp. 933-940, Nov. 1997.

[10] "Direct Digital Synthesizer (DDS) V4.0 Product Specification," Xilinx Inc., San Jose, CA, Oct. 2001.

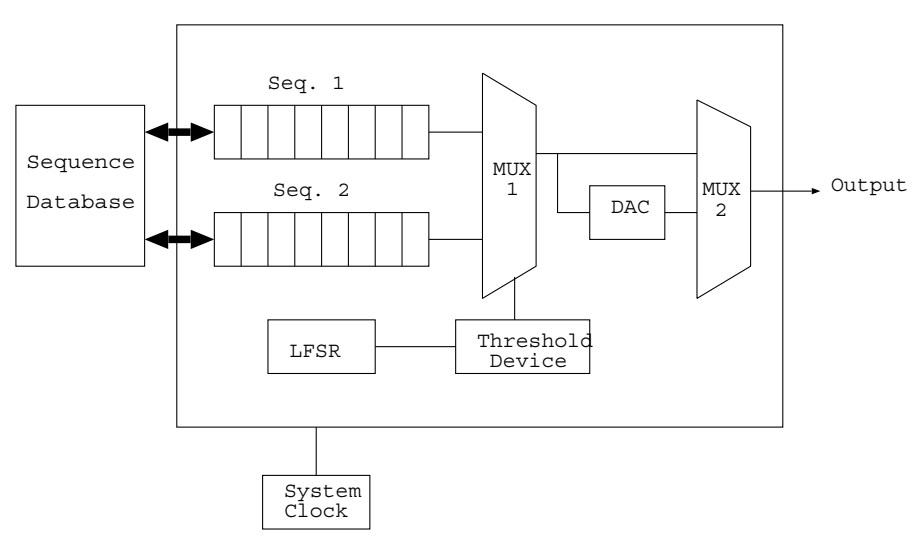

Fig. 1. System blocks of IS.

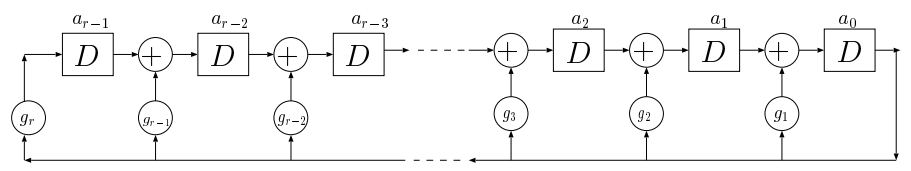

Fig. 2. LFSR of Galois configuration [6].

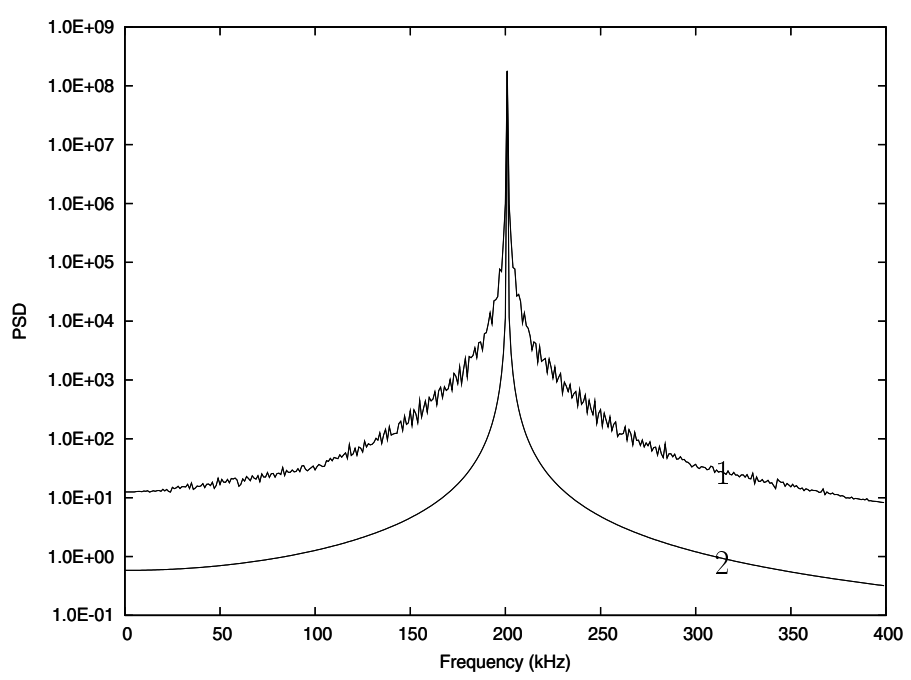

Fig. 3. PSDs of the synthesized signal and its corresponding exact signal. Curve 1: Synthesized signal using IS. Curve 2: Corresponding exact signal 


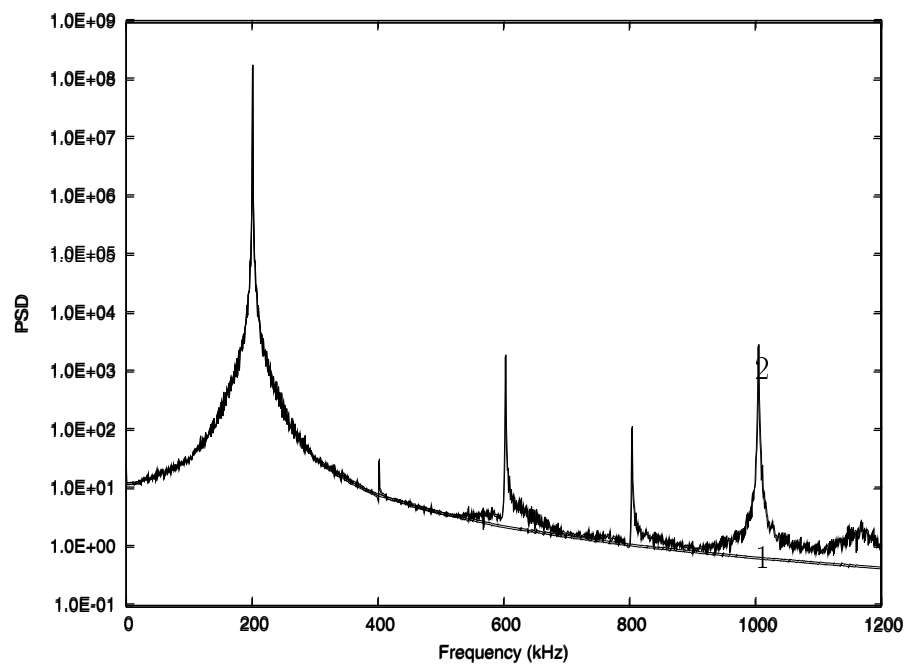

Fig. 4. Effects of finite-precision on PSDs. Curve 1: Floating-point numerical format. Curve 2: Fixed-point numerical format with 5 bits per sample. 